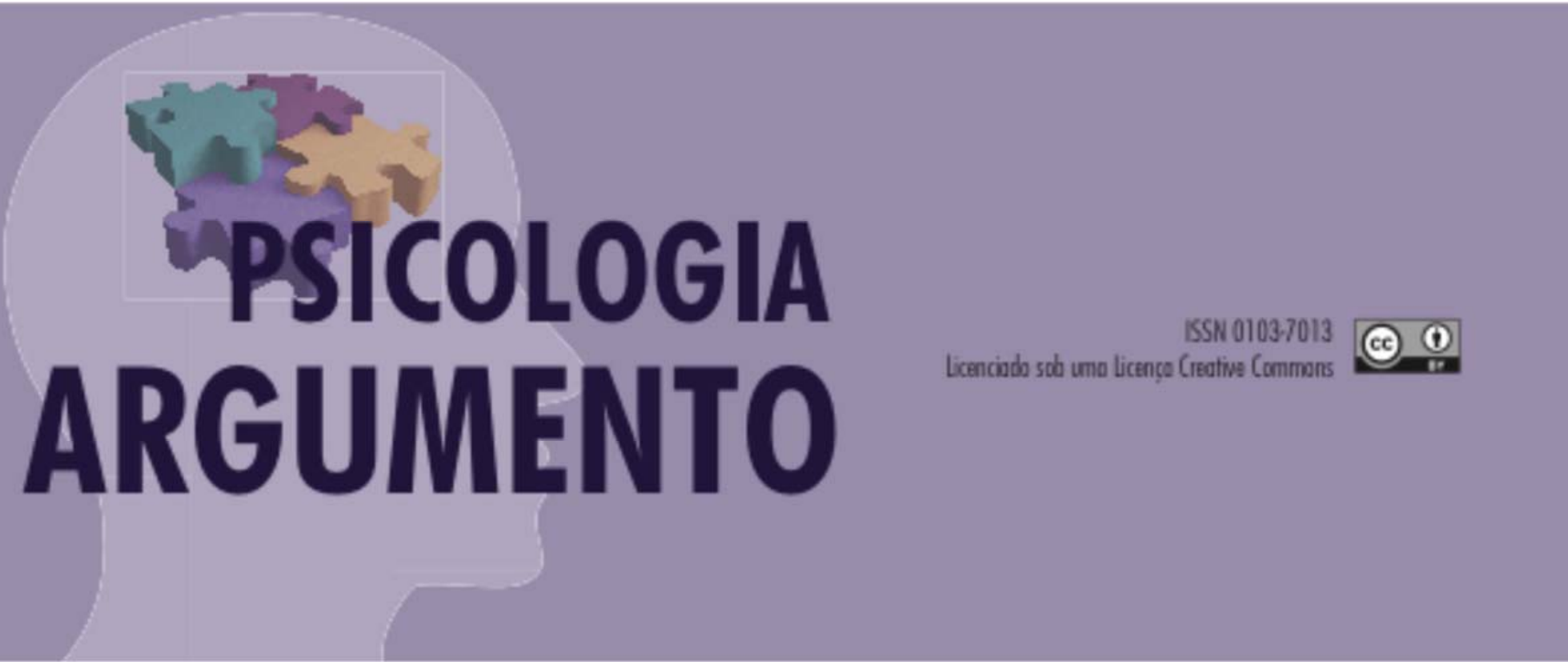

doi: http://dx.doi.org/10.7213/psicolargum.36.91.AO05

\title{
Técnicas de entrevista em casos de abuso sexual infantojuvenil: uma revisão sistemática.
}

Interview techniques in cases of child sexual abuse: a systematic review.

VIVIAN SENEGALIA MORETE

Mestra, Analista Judiciária - área Psicologia, Tribunal de Justiça do Estado do Paraná, TJPR, Av. Duque de Caxias, nº 689, CEP: 86015-000, Londrina, Paraná, Brasil. E-mail: vsmo@tjpr.jus.br - autor para correspondência.

ALEX EDUARDO GALLO

Doutor, Professor, Universidade Estadual de Londrina, UEL, Londrina, Paraná, Brasil. E-mail: aedgallo@uel.br.

GIOVANA VELOSO MUNHOZ DA ROCHA

Doutora, Professora, Universidade Tuiuti do Paraná, UTP, Curitiba, Paraná, Brasil. Email: gimunhozdarocha@gmail.com.

\section{Resumo}

Neste artigo apresenta-se uma revisão sistemática da produção científica empírica sobre técnicas de entrevista para investigação de abuso sexual infantojuvenil. Foram feitas buscas sobre o tema em sete bases de dados eletrônicas e recuperados 54 títulos entre artigos, teses e dissertações. Observou-se a existência de um contínuo de risco, em que as questões abertas são as que menos geraram contradições e mais favoreceram a descrição da suposta situação abusiva e as questões múltipla-escolha e sugestivas as que menos contribuíram com a fidedignidade dos relatos. Discutiu-se sobre o uso de protocolos de entrevistas, de entrevistas não estruturadas, de recursos adicionais às entrevistas, do 
estabelecimento de rapport e outros. Ao final foram delineadas direções para investigação científica.

Palavras-chave: abuso sexual, avaliação forense, entrevista, Psicologia.

\begin{abstract}
This paper presents a systematic review of empirical publications about interview techniques used to investigate child sexual abuse. We searched seven database and recovered 54 papers, thesis and dissertations. We found a risk continnum where open questions produced fewer contradictions and supported the description of alleged abusive situation and multiple-choice questions were less supportive to the trust of report. We discussed the use of interview protocols, non-structured interviews, and use of additional resources, rapport settings and others. At the end, we designed guidelines for scientific research.
\end{abstract}

Keywords: sexual abuse, forensic evaluation, interview, psychology.

\title{
Resumen
}

En este artículo se presenta una revisión sistemática de la producción científica empírica sobre las técnicas de entrevista para la investigación del abuso sexual infanto juvenil. Se realizaron búsquedas sobre el tema en siete bases de datos electrónicas y fueron recuperados 54 títulos entre artículos, tesis y disertaciones. Se observó la existencia de un continuo de riesgo, en que las cuestiones abiertas son las que menos generaron contradicciones y más favorecieron a la descripción de la supuesta situación abusiva y las cuestiones múltiple-elección y sugestivas fueron las que menos contribuyeron con la fidedignidad de los relatos. Se discutió sobre el uso de protocolos de entrevistas, de entrevistas no estructuradas, de recursos adicionales a las entrevistas, del establecimiento de rapport y otros. Al final se delinearon direcciones para la investigación científica.

Palabras clave: abuso sexual, evaluación forense, entrevista, Psicología..

\section{Introdução}

O enfrentamento ao abuso sexual infantojuvenil tem sido objeto de crescente investimento em mobilização e planejamento governamental e social nas últimas décadas. No Brasil, um dos marcos deste investimento, foi a consolidação da proposta de elaboração de um Plano Nacional de Enfrentamento da Violência Sexual Infantojuvenil, cujas ações deverão ser implementadas até 2020 (Plano Nacional de Enfrentamento, 2013).

Seguindo as diretrizes internacionais formuladas no III Congresso Mundial de Enfrentamento da Exploração Sexual de Crianças e Adolescentes, realizado em 2008 no Rio de Janeiro-BR, o Plano Nacional destaca a violência sexual infantojuvenil como sendo "todo ato, de qualquer natureza, atentatório ao direito humano ao desenvolvimento sexual da criança e do adolescente, praticado por agente em situação de poder e de desenvolvimento sexual desigual em relação à criança e adolescente vítimas." (Plano Nacional de Enfrentamento, 2013, p. 22), conceito que se alinha com o da Organização Mundial de Saúde (World Health Organization - WHO, 1999; Brino, 2007). 
A constatação de que crimes dessa natureza ocorrem preponderantemente no âmbito privado, não resultam necessariamente em vestígios físicos e são presenciados, muitas vezes, exclusivamente por suas vítimas e agressores, implica em desafios para a obtenção de provas e na conferência de especial relevância ao relato verbal da criança ou adolescente (Gava, Pelisoli, \& Dell'Aglio, 2013; Schaefer, Rossetto, \& Kristensen, 2012). A entrevista com as supostas vítimas pode ser realizada na fase de inquérito policial, quando denominada entrevista investigativa e que não necessariamente é conduzida por psicólogos (Souza, 2014); ou ser realizada em contexto judicial quando, então, é denominada entrevista forense, usualmente um dos instrumentos do psicólogo na realização de perícias.

Segundo a American Professional Society on the Abuse of Children (APSAC), o objetivo da entrevista forense é obter um relato o mais acurado e completo possível acerca do suposto abuso com o intuito de determinar se a criança ou adolescente sofreu de fato a violência ou está em iminente risco de sofrer e, em caso afirmativo, por quem (Cronch, Viljoen, \& Hansen, 2006). O escopo da entrevista forense é, portanto, coletar informações relevantes para compreender um evento cujo esclarecimento favorecerá uma medida de proteção da criança e a responsabilização criminal do agressor (Williams, Hackbarth, Blefari, Padilha, \& Peixoto, 2014).

É imprescindível que a atuação do profissional encarregado de conduzir entrevistas e intervenções com supostas vítimas seja baseada em evidências de pesquisas acerca de técnicas que favoreçam a descrição pela criança do que de fato ela vivenciou, com o mínimo de indução possível. Entrevistas mal conduzidas podem gerar consequências graves como levantar falsas alegações, expor as crianças e suas famílias a condições desnecessárias de estresse, desacreditar o relato da suposta vítima perante o juízo, induzir relatos imprecisos sobre os fatos, reduzir a probabilidade de condenação, entre outros (Cronch et al., 2006).

As questões iniciais que se impõem, portanto, são: quais técnicas de entrevista psicológica para avaliação de abuso sexual infantojuvenil têm sido objeto de pesquisa? O que os resultados das pesquisas que avaliam essas técnicas têm a informar aos prestadores de serviço na área da proteção à infância e juventude? O presente trabalho objetivou revisar sistematicamente a produção científica empírica sobre técnicas de entrevista em casos de suspeita de abuso sexual infantojuvenil.

\section{Método}

Para a concretização da pesquisa foi utilizada a metodologia da revisão sistemática que "se refere ao processo de reunião, avaliação crítica e sintética de resultados de múltiplos estudos" (Costa \& Zoltowski, 2014). As palavras-chave utilizadas para as buscas foram: abuso sexual infantil, entrevista psicológica e entrevista investigativa. Os termos foram pesquisados em português e inglês. As combinações de palavras usadas foram: abuso sexual infantil e entrevista psicológica; abuso sexual infantil e entrevista investigativa. A busca e armazenamento dos resultados ocorreram na primeira semana de fevereiro de 
2015. Procedeu-se à busca nas seguintes bases de dados: Psyclnfo, Web of Science, IndexPsi, Scielo, Google Acadêmico, Portal Domínio Público de Teses e Dissertações da CAPES e Biblioteca Digital de Teses e Dissertações - BDTD.

A literatura obtida a partir dessa pesquisa foi selecionada mediante aplicação de alguns critérios. Os critérios de inclusão foram: a) trabalhos empíricos cujo tema central era a entrevista psicológica ou investigativa em casos de suspeita de abuso sexual contra crianças e adolescentes; b) que envolviam a análise da estrutura/fases da entrevista, recursos suplementares e tipos de perguntas utilizados; c) que abordavam a influência do entrevistador no relato de crianças e adolescentes supostamente vítimas; d) estudos comparativos das técnicas de entrevista; e e) publicados entre os anos de janeiro de 2000 e janeiro de 2015. Foram excluídos os trabalhos: a) repetidos ou incompletos; b) sobre abusadores; c) sobre o perfil ou dinâmica familiar frente ao relato de abuso pela criança; d) sobre os efeitos psicológicos do abuso para a criança; e) a respeito de psicoterapia com crianças ou adolescentes vítimas de violência sexual; f) sobre características das crianças envolvidas nas denúncias; e g) estudos focados em treinamentos de profissionais em técnicas específicas.

Do total de 510 referências potencialmente relevantes localizadas, foram recuperadas 54 produções científicas que atenderam aos critérios supramencionados.

\section{Resultados e Discussão}

Os estudos empíricos sobre entrevista em casos de abuso sexual infantojuvenil foram classificados a fim de favorecer sua análise e discussão. Uma primeira classificação geral se referiu ao tipo de estudo realizado: a) estudos com situações reais de denúncia de abuso sexual infantojuvenil; ou b) estudos análogos, nos quais são construídos contextos que permitem a análise de variáveis semelhantes àquelas presentes em situações de investigação de abuso sexual (passagem de tempo, toque corporal, questionamentos sugestivos, entre outros), porém, com controle da situação vivenciada pela criança, o que favorece o estudo da precisão/acurácia do relato infantil (Poople \& Bruck, 2012).

Dos estudos recuperados, $44(81,5 \%)$, envolveram situações reais de denúncia de abuso e 10 (18,5\%), consistiram em estudos análogos. Cada tipo de estudo apresenta vantagens e desvantagens. Estudos análogos, embora dificilmente consigam simular os fatores emocionais a que as vítimas são submetidas, como, por exemplo, a significativa ameaça de punição, sentimentos de culpa e vergonha, efeitos de estresse pós-traumático, entre outros, viabilizam análise precisa de como o tipo de enunciado utilizado pelo entrevistador relacionase com relatos imprecisos ou fantasiosos das crianças/adolescentes. Dentre os estudos análogos, destacaram-se os estudos desenvolvidos em contextos médicos (Lyon, 2012).

Os estudos de campo, por sua vez, contam com a vantagem de possuir validade externa e, portanto, proporcionam resultados mais generalizáveis à população. Tais estudos viabilizam o desenvolvimento de alternativas para lidar com crianças sob vários tipos de contingências aversivas e, por vezes, relutantes 
em falar sobre suas vivências. Como ponto de dificuldade, contudo, destaca-se que muitas vezes não é viável nesses estudos analisar a precisão do relato da criança. Nesta pesquisa, dentre os estudos com situações reais de denúncia recuperados, em apenas três foi possível que os autores aferissem, de forma indireta, sobre a acurácia dos relatos infantis mediante comparação entre os dados das entrevistas e a obtenção de outros meios substanciais de provas (e.g. Hershkowitz, Fisher, Lamb, \& Horowitz, 2007; Hershkowitz, Orbach, Lamb, Sternberg, \& Horowitz, 2006) ou checagem dos relatos das crianças com informações sobre a condenação dos acusados (e.g. Schreiber, 2000). Outra estratégia utilizada pelos pesquisadores de campo para obter informações referentes à acurácia dos relatos das crianças em alguns dos estudos recuperados, foi a análise de contradições internas, ou seja, contradições existentes em uma ou em mais de uma entrevista com a mesma criança (e.g. Katz \& Hershkowitz, 2013; Lamb \& Fauchier, 2001; Orbach \& Lamb, 2001) ou a utilização do CBCA (CriteriaBased Content Analysis), ferramenta validada empiricamente para avaliar a credibilidade do relato das crianças e que auxilia na discriminação entre alegações plausíveis ou não (e.g. Daviesl, Westcott, \& Horan, 2000; Schreiber, 2000).

No que diz respeito ao conteúdo de análise dos estudos, segunda classificação utilizada, destacaram-se as seguintes categorias: 1) entrevistas com o uso de protocolos padronizados: 1.1) entrevistas com o uso do protocolo NICHD; 1.2) entrevistas baseadas no Memorandum of Good Practice ou no seu sucessor, o Achieving Best Evidence in Criminal Proceedings - Guidance on Interviewing Victims and witnesses, and Using Special Measure; 1.3) entrevistas com outros protocolos; 2) entrevistas não estruturadas ou com técnicas não informadas; 3) uso do diagrama corporal como técnica associada à entrevista; 4) uso de desenho como técnica associada à entrevista; 5) entrevistas com técnicas cognitivas; 6) outros.

\section{Entrevistas com o uso de instrumentos padronizados.}

\subsection{Entrevistas com o uso do protocolo NICHD.}

A técnica de entrevista mais pesquisada nos estudos recuperados foi o protocolo NICHD (mais de 35\% dos estudos), desenvolvido pelo National Institute of Child Health and Human Development, cuja sigla dá nome ao protocolo.

Nas pesquisas com o protocolo NICHD foram observados alguns conteúdos específicos de investigação. O primeiro deles diz respeito aos estudos sobre a fase inicial da entrevista, denominada pré-substantiva, com destaque à construção de rapport e à prática narrativa. O segundo conteúdo analisado foi o efeito do tipo de enunciado formulado pelo entrevistador sobre a resposta dos entrevistados e, por fim, o efeito da repetição de entrevistas e do gênero do entrevistador.

Fase pré-substantiva no protocolo NICHD.

Três pesquisas referenciadas analisaram a fase pré-substantiva do protocolo NICHD de forma específica (Ahern, Hershkowitz, Lamb, Blasbalg, \& Winstanley, 
2014; Brown, Lamb, Lewis, Pipe, Orbach, \& Wolfman, 2013; Hershkowitz et al., 2006).

Tais estudos apontam que a forma como a fase pré-substantiva é conduzida interfere nos resultados da etapa subsequente do protocolo, que é específica sobre o suposto abuso. Deste modo, há que se atentar para a condução do rapport com prática narrativa e com comentários de suporte contingentes às respostas de resistência dos entrevistados, o que tende a favorecer a cooperação e a precisão dos relatos (Ahern, Hershkowitz, Lamb, Blasbalg, \& Winstanley, 2014; Brown, Lamb, Lewis, Pipe, Orbach, \& Wolfman, 2013; Hershkowitz et al., 2006). Embora esses estudos sejam pontuais e haja ressalvas quanto à possibilidade de generalização, observar-se-á que, em estudos com outros padrões de entrevista, descritos posteriormente, há resultados que reforçam essas mesmas orientações.

Relação entre tipos de enunciados do protocolo NICHD e a resposta dos entrevistados.

Os seis estudos recuperados que tratam da relação entre os tipos de enunciados (e.g. Lamb, Hershkowitz, Sternberg, Esplin, Hovav, Manor, \& Yudilevitch, 1996; e revisado por Lamb, Hershkowitz, Orbach, \& Esplin, 2008) e as respostas dos entrevistados, avaliaram, juntos, 468 entrevistas com crianças e adolescentes supostamente vítimas de violência física ou sexual, em sua maioria na faixa entre 4 e 13 anos (Hershkowitz, 2002; Katz \& Hershkowitz, 2012; Lamb, Stemberg, \& Orbach, 2003; Orbach \& Lamb, 2000; Orbach, Hershkowitz, \& Lamb, 2000; Sternberg, Lamb, Orbach, Esplin, \& Mitchell, 2001).

No que diz respeito à efetividade do uso de facilitadores - uma das categorias de enunciados, que incluem falas "como 'OK', reformulações do que foi dito anteriormente pela criança e palavras de encorajamento (não sugestivas) voltadas a manter a criança falando" (Blefari, 2014, p. 50) - a depender da fase da entrevista e de sua posição em relação a outros enunciados, observou-se que diante de cada facilitador a criança apresentou, em média, cinco novos detalhes substanciais. Os facilitadores apresentados seguidamente às solicitações proporcionaram maior número de palavras e detalhes quando comparado aos outros tipos de enunciados. Além disso, os facilitadores foram mais efetivos na primeira parte da fase substantiva da entrevista (etapa em que usualmente a criança apresenta relatos mais longos e ricos) (Hershkowitz, 2002).

Em estudos que compararam entrevistas conduzidas com e sem o uso do protocolo NICHD (Orbach et al., 2000; Sternberg et al., 2001) as entrevistas com o protocolo contiveram mais solicitações/questões abertas e esse tipo de enunciado foi mais utilizado antes de questões de múltipla-escolha se comparado à condição controle. Com o protocolo, houve maior investimento na parte pré-substantiva e menos detalhes foram obtidos com 0 uso de solicitações sugestivas, independentemente da idade dos entrevistados (Orbach et al., 2000).

Paralelamente à categorização descrita anteriormente, um dos estudos avaliou a frequência e os efeitos do uso de questões múltiplas durante entrevistas investigativas de abuso sexual com o protocolo NICHD (Katz \& Hershkowitz, 2012). 
A maioria das crianças, independentemente de idade, respondeu a apenas uma pergunta, ou, em segundo lugar, forneceram respostas não inteligíveis. As respostas às questões múltiplas, em sua maioria, foram mais curtas e continham menos detalhes se comparadas às respostas dadas às questões simples (Katz \& Hershkowitz, 2012).

Efeitos da repetição de entrevista com o protocolo NICHD.

Discute-se que um procedimento longo para avaliação de abuso sexual infantil pode expor a criança a risco uma vez que ela retornaria ao ambiente familiar, que não se sabe se é protetor, e esperaria para ser atendida novamente, permanecendo vulnerável a posteriores abusos. Além disso, pesquisas demonstraram que a realização de mais de uma entrevista pode levar a distorções nos relatos, aumentar a probabilidade de contradições e tornar a situação mais angustiante para a criança. $O$ ideal, nesse sentido, seria que a revelação fosse viabilizada com uma única entrevista (Cronch et al., 2006). Contudo, múltiplas entrevistas podem ser necessárias em alguns casos uma vez que as crianças e adolescentes podem se sentir desconfortáveis em expor a situação abusiva a um desconhecido, necessitar de tempo para estabelecimento de rapport e possuírem capacidade restrita de concentração (especialmente as menores) (Cronch et al., 2006).

Dentre as pesquisas recuperadas, duas avaliaram a realização de múltiplas entrevistas com o protocolo NICHD (Hershkowitz \& Terner, 2007; Katz \& Hershkowitz, 2013). Entretanto, as entrevistas ocorriam em intervalos muito breves de tempo, poucos minutos (30 min e $7 \mathrm{~min}$ ), o que poderia ser considerado efetivamente um intervalo não fosse a solicitação para que as crianças/adolescentes dissessem novamente o que lhes havia acontecido, como se não tivessem feito isso anteriormente. Ambas as pesquisas foram conduzidas em Israel, uma analisou 40 entrevistas, com crianças entre 6 e 13 anos (Hershkowitz \& Terner, 2007) e a outra analisou entrevistas conduzidas com 56 crianças e adolescentes, de 5 a 14 anos (Katz \& Hershkowitz, 2013). Houve acréscimo significativo de informações forensicamente relevantes na segunda entrevista, sendo que grande parte delas diziam respeito a detalhes centrais da situação (Katz \& Hershkowitz, 2013; Hershkowitz \& Terner, 2007).

Influência do gênero do entrevistador - comparação do protocolo NICHD com outros modelos de entrevista.

Houve uma pesquisa, com análise de 672 entrevistas, provenientes de Israel, Estados Unidos e Grã-Bretanha, com crianças, entre 4 e 14 anos, supostamente vítimas de abuso sexual, que demonstrou efeito significativo do gênero tanto no comportamento do entrevistador quanto no volume de informações fornecidas pelas crianças. Mulheres utilizaram mais solicitações e questões sugestivas com as crianças do sexo masculino, do que com crianças do sexo feminino. Homens 
entrevistaram crianças de ambos os sexos de forma similar. Meninas, de todas as idades, forneceram mais informações em resposta às solicitações diretivas feitas por mulheres. O gênero do entrevistador não resultou em efeitos significativos nas respostas das crianças do sexo masculino. Mais informações foram obtidas de crianças menores em resposta a solicitações sugestivas de entrevistadores do sexo oposto. As questões de gênero foram atenuadas com o uso de protocolos (Lamb \& Garretson, 2003).

\subsection{Memorandum of Good Practice e sua versão atualizada, o Achieving Best Evidence in Criminal Proceedings - Guidance on Interviewing Victims and witnesses, and Using Special Measure}

Dentre as pesquisas recuperadas nesta revisão, duas envolveram explicitamente o Memorandum (Daviesl et al., 2000; Lamb, Orbach, \& Sternberg, 2009) e duas foram conduzidas em serviços do País de Gales e da Inglaterra após a edição do Memorando, portanto, pressupõe-se que analisaram resultados de entrevistas baseadas neste documento (Phillips, Oxburgh, Gavin, \& Myklebust, 2012; Westcott e Kynan, 2004). Mediante análise conjunta das pesquisas com o Memorando - considerando que a quantidade de questões abertas ou apropriadas não consistiu o tipo de questão mais formulado pelos entrevistadores sempre que as orientações deste guia foram seguidas - ponderou-se que o uso de um protocolo estruturado seria preferível, favorecendo maior adesão às orientações de boas práticas em entrevistas com crianças. Também se observou que a quantidade e a qualidade de informações obtidas em entrevistas são influenciadas de forma significativa pelo tipo de pergunta e pelo estilo do entrevistador.

\subsection{Entrevistas com outros protocolos.}

A partir das pesquisas descritas, observa-se que, de 2000 a 2015, intervalo utilizado para a busca de literatura nesta revisão, poucos foram os estudos que se dedicaram à análise experimental ou empírica de outros protocolos de entrevista que não o protocolo NICHD. Esses estudos analisaram protocolos como o RATAC, a Yuille Step-wise interview e outros protocolos sem denominações específicas, desenvolvidos pelos pesquisadores (Anderson, Anderson, \& Gilgun, 2014; Feltis, Powell, Snow, \& Hughes-Scholes, 2010; Lindberg, Chapman, Samsock, Thomas, \& Lindberg, 2003). Destaca-se, contudo, que os resultados dos estudos corroboram resultados de pesquisas com o protocolo NICHD, de que questões abertas e facilitadores favorecem relatos mais detalhados pelas crianças e 
adolescentes e que questões diretivas - "falas do entrevistador que focam em um detalhe sobre o incidente mencionado pela criança anteriormente na entrevista e solicitam uma informação adicional utilizando normalmente perguntas "wh" (Quem, O que?, Qual? Onde? Quando? Como?)" (Blefari, 2014, p. 53) - se comparadas às questões abertas, aumentam a probabilidade deles se reportarem a fatos não ocorridos.

\section{Entrevistas não estruturadas ou com técnicas não informadas.}

As pesquisas acerca de métodos de entrevista não estruturados ou cujas técnicas não constam precisamente descritas no procedimento dos artigos, foram desenvolvidas em mais de cinco países, entre eles Austrália, Malásia, Brasil, Estados Unidos, Finlândia, Nova Zelândia, Estônia e Suécia, totalizando 717 entrevistas de investigação de suspeita de abuso sexual infantojuvenil analisadas (Brubacher \& La Rooy, 2014; Cederborg, Danielsson, La Rooy, \& Lamb, 2009; Cheung, 2008; Fogarty, Augoustinos, \& Kettler, 2013; Gilstrap, 2004; Kask, 2012; Korkman, Santtila, \& Sandnabba, 2006; Patterson \& Pipeb, 2009; Santila, Korkman, \& Sandnabba, 2004; Schreiber, 2000; Snow, Powell, \& Murfett, 2009; Stracke, 2013; Teoh \& Lamb, 2010). Tratam-se de dezenove estudos (35\% da amostra) que procuraram analisar desde a relação entre tipos de enunciados e a resposta dos entrevistados, até os efeitos da repetição de entrevistas na qualidade e volume de informações prestadas, e o rapport.

Relação entre tipos de enunciados de entrevistas não estruturadas e a resposta dos entrevistados.

Assim como diversas pesquisas recuperadas avaliaram a relação entre o comportamento do entrevistador e a resposta das crianças/adolescentes com o uso do protocolo NICHD, montante significativo dos estudos - aproximadamente 9\% da amostra - envolveu a análise dessa mesma relação, porém, com o uso de métodos de entrevista ordinariamente utilizados em diversas localidades, mas não formalizados em um protocolo. Nessas circunstâncias em que as orientações, se existentes, não eram formalizadas em um documento específico, observou-se que a maior parte das questões usualmente apresentadas pelos entrevistadores tendeu a ser diretiva (que se atentam a detalhes anteriormente mencionados pela criança e usualmente são iniciadas com "o que", "quando", "onde", por exemplo) ou de múltipla-escolha (Kask, 2012; Korkman et al., 2006; Korkman, Santtila, Westeraker, 
M., \& Sandnabbaa, 2008), em que pese as questões abertas tenham produzido volume significativamente maior de informações (Kask, 2012).

O maior número de detalhes foi obtido a partir de solicitações diretivas, possivelmente porque foi um dos tipos de questões mais utilizado; seguidas pelas questões abertas, que mesmo consistindo em enunciado muito pouco apresentado (algumas vezes apenas $2 \%$ dos enunciados formulados pelos entrevistadores), ainda assim destacaram-se na produção de informações relevantes (Daviesl et al., 2000). Depois das crianças apresentarem detalhes, os entrevistadores, de modo geral, apresentaram mais facilitadores e solicitações diretivas. A categoria das crianças que esteve relacionada ao aumento do número de questões abertas pelo entrevistador, foi a de respostas "sim e não" (Korkman et al., 2006). Na pesquisa de Cheung (2008) constatou-se que nas entrevistas em que houve revelação de abuso foram utilizadas mais perguntas iniciadas com "o que" e "como". O uso de questões muito longas também resultou em respostas breves na pesquisa de Korkman et al. (2008), na qual observou-se igualmente que questões breves repercutiram em respostas mais descritivas.

Nos resultados das duas pesquisas publicadas em 2001, observou-se uma predominância da relação entre questões sugestivas e de múltipla escolha com as contradições dos relatos das crianças, que se contradisseram inclusive quanto aos aspectos mais importantes da investigação (Lamb \& Fauchier, 2001; Orbach e Lamb, 2001). Além disso, quanto mais repetições das perguntas, maior probabilidade de modificação da resposta foi constatada em outro estudo (Cederborg et al., 2009).

A única pesquisa brasileira levantada na amostra foi a de Stracke (2013) sobre a avaliação da estrutura de entrevista investigativa com crianças, que analisou entrevistas conduzidas por um serviço do Rio Grande do Sul -BR. Utilizando-se categorizações pré-existentes e metodologia bastante semelhantes aos estudos sobre o protocolo NICHD descritos anteriormente, fez-se a codificação de perguntas e respostas constantes das fases pré-substantiva e substantiva das entrevistas. As questões de sondagem, cuja descrição equivale às diretivas de outros estudos - e.g. onde vocês estavam quando isso aconteceu? - ou fechadas apropriadas - e.g. isso que me contou, aconteceu mais de uma vez? - foram as mais relacionadas a respostas extensas pelas crianças. Houve também significativa associação entre as respostas curtas e as questões fechadas apropriadas. O resultado deste estudo contrasta com estudos conduzidos por outros pesquisadores já que questões fechadas apropriadas, e não as questões abertas, produziram respostas mais extensas das crianças. 
Analisando-se as pesquisas que tratam da relação entre tipos de enunciados e a resposta dos entrevistados em entrevistas não estruturadas, podem ser salientados alguns pontos: a) dado o número de pesquisas neste subitem, observa-se volume significativo de localidades que não seguem qualquer tipo de protocolo ou guia para a condução de entrevistas; b) quando a entrevista não é estruturada, há alta taxa de enunciados diretivos ou de múltipla-escolha; e c) mesmo tendo sido baixas as taxas de convites/questões abertas nas entrevistas não estruturadas, este tipo de enunciado foi aquele que mais favoreceu relatos extensos e não contraditórios pelas crianças.

Estudos sobre rapport em entrevistas não estruturadas.

O rapport nas entrevistas não estruturadas foi objeto de dois estudos com concepções teóricas distintas. Fogarty et al. (2013), exploraram como a análise da "progressividade da conversação" poderia proporcionar tratabilidade empírica para o conceito de rapport e sugerir novas direções de pesquisa e treinamento com relação às entrevistas investigativas com crianças supostamente vítimas de violência.

Progressividade da conversação é um conceito que significa evolução, durante uma determinada sequência/alternância entre os interlocutores, de como as ações destes colaboram com o desenvolvimento/continuidade da interação/atividade. Fogarty et al. (2013), baseando-se neste conceito, partiram do pressuposto de que o rapport não constitui um estado fixo, e, portanto, passível de se circunscrever em uma etapa da entrevista, mas, ao contrário, trata-se de fluxo que ocorre durante toda a interação entre entrevistador e entrevistado. Para avaliar o estabelecimento de rapport, em compreensão compatível com a visão da Análise do Comportamento, não fizeram inferências sobre o estado emocional das crianças entrevistadas. Avaliaram as respostas observáveis da criança para efetuar esta análise, tais como: contato visual, sorriso, movimentos da cabeça, entre outros. Observaram que o olhar pode ser um dos correlatos não verbais do rapport, vez que havia prejuízo à sequência e desenvolvimento da entrevista - à progressividade - quando o olhar da criança não se dirigia ao entrevistador. Contudo, assim como outros correlatos não-verbais tais como sorrir ou acenar com a cabeça, o olhar fixo ou outros comportamentos, apenas relacionaram-se ao rapport quando apresentados em momentos específicos da interação, não estando genericamente relacionados ao desenvolvimento da interação ou a sentimentos de simpatia ou afetividade (Fogarty et al., 2013).

O outro estudo sobre a fase pré-substantiva, desenvolvido por Teoh e Lamb (2010), enfatizou a construção do rapport em uma etapa preparatória para a 
entrevista investigativa, o que foi mais comum se considerada a amostra recuperada nesta revisão bibliográfica. Com crianças pequenas, os entrevistadores utilizaram mais enunciados de construção de rapport e despenderam mais tempo nesta etapa. Quão mais longos foram os períodos para o rapport, menos informativas foram as crianças pequenas na fase substantiva (Teoh \& Lamb, 2010).

Efeitos da repetição de entrevistas não estruturadas.

Das pesquisas recuperadas, quatro trataram do tema da repetição de entrevistas sem o uso de protocolos (Brubacher \& La Rooy, 2014; Patterson \& Pipeb, 2009; Santila, Korkman, \& Sandnabba, 2004; Schreiber, 2000). Observouse que a repetição de entrevistas em formato não estruturado possivelmente afetou de forma significativa os resultados obtidos. Assim como ocorre com entrevistas não estruturadas isoladamente, houve maior probabilidade do entrevistador não seguir orientações de boas práticas, como iniciar solicitando descrição geral e, somente após, transcorrer para a individualização de episódios; e evitar questões sugestivas e de múltipla-escolha. Diante desses elementos, constatou-se que a repetição de entrevistas não estruturadas pode comprometer a fidedignidade dos relatos e ainda demanda mais investigação científica.

\section{Uso do diagrama corporal como técnica associada à entrevista.}

O diagrama corporal tem sido utilizado de várias formas em entrevistas investigativas de abuso sexual infantil por acreditar-se que seu uso favoreça a revelação do abuso sexual pela criança. Alguns profissionais utilizam o diagrama para introduzir a discussão sobre toques inapropriados e, outros, inserem o diagrama em fases posteriores da entrevista, a fim de obter esclarecimentos sobre toques descritos anteriormente pela criança. Este último uso do diagrama corporal tem sido mais indicado por pesquisadores por tratar-se de formato menos sugestionável (Poole \& Dickinson, 2011).

Aldridge, Lamb, Sternberg, Orbach, Esplin e Bowler (2004) e Teoh, Yang, Lamb, e Larsson (2010) avaliaram o uso de diagramas não anatomicamente detalhados, apresentados às crianças após entrevista com o uso do protocolo NICHD. O diagrama auxiliou os investigadores a obterem informação significativa para o contexto forense mesmo após extensiva entrevista com a criança, tendo sido especialmente útil com as crianças de 4 a 7 anos. Durante a utilização do recurso diagrama, os entrevistadores fizeram uso frequente de questões de 
múltipla escolha - que trabalha com a memória de reconhecimento - o que pode repercutir em menor fidedignidade das informações (Aldridge et al., 2004).

O uso do diagrama foi associado a relatos de toques não mencionados pelas crianças durante a entrevista com o protocolo NICHD. Também houve elaborações sobre as partes do corpo em que havia sido reportado toque durante a entrevista. Embora as crianças, de modo geral, tenham reportado mais detalhes durante a entrevista, após a apresentação do desenho, houve aumento do número de detalhes claros sobre os toques, com nomeação correta das partes do corpo e das ações ocorridas, especialmente por crianças mais velhas (Teoh et al., 2010).

Em estudo desenvolvido por Poole e Dickinson (2011), contudo, os resultados não foram tão favoráveis à utilização do recurso. Quatorze vírgula cinco por cento das crianças que passaram pela entrevista com o diagrama reportaram toques que nunca ocorreram, o que não aconteceu com os grupos que passaram pela entrevista padrão. Os autores sugerem que, caso utilizados, os diagramas sejam introduzidos em fase mais avançada da entrevista, após etapa com amplo uso de questões abertas.

\section{Uso do desenho como ferramenta associada à entrevista.}

O desenho tem sido considerado instrumento útil à construção de rapport por tratar-se de atividade rotineira e lúdica usualmente realizada por crianças em outros contextos. O desenho também é utilizado como recurso complementar às entrevistas com crianças com dificuldade de expressão através da fala, seja por estarem desenvolvendo esta habilidade, seja por questões de deficiência motora, mental ou outras. Uma terceira justificativa de pesquisadores para a associação entre desenho e entrevista investigativa, é que o desenho pode ser útil para a autogeração de dicas contextuais que favorecem o relato infantil sobre a suposta experiência de abuso (Katz \& Hershkowitz, 2010).

Os estudos recuperados que versam sobre o uso do desenho, foram todos desenvolvidos associando este recurso à entrevista baseada no protocolo NICHD (Katz, Barnetz, \& Hershkowitz, 2014; Katz e Hamama, 2013; Katz e Hershkowitz, 2010). Em todos os estudos houve entrevista inicial com o protocolo NICHD e, após, era solicitado que a criança desenhasse o que havia the ocorrido e contasse novamente a situação do início ao fim. Note-se que não há interpretação do desenho pelo entrevistador, este é utilizado como instrumento facilitador e organizador do relato da criança, este sim empregado para fins probatórios. Após a confecção dos desenhos, as crianças relataram as situações abusivas de forma mais sequencial e organizada, além de terem fornecido mais informações 
relevantes (Katz \& Hamama, 2013). Foi observado que, diante de questões abertas, houve fornecimento de mais detalhes pelo grupo da condição de desenho; não tendo sido demonstrada diferença significativa entre os grupos diante de questões focadas (Katz \& Hershkowitz, 2010).

\section{Entrevistas com técnicas cognitivas.}

Os dados foram contraditórios, vez que no primeiro estudo não foram observados benefícios e, no segundo, observou-se que o restabelecimento mental de contexto favoreceu as respostas às questões abertas e não foi menos efetivo do que o restabelecimento físico de contexto (Darwinkel, Powell, \& Sharman, 2014; Hershkowitz, Orbach, Lamb, Sternberg, \& Horowitz, 2002). Investigações na linha do estudo de Hershkowitz et al. (2002) possuem maior validade externa por terem ocorrido em campo e por ter contado com maior número de participantes. Destaque-se que, caso haja futura corroboração dos resultados deste estudo, a incorporação do restabelecimento mental de contexto em entrevistas forenses é viável do ponto de vista prático, não importando em custos adicionais de deslocamento ou organização logística.

\section{Outros estudos recuperados.}

Outras duas pesquisas recuperadas foram exclusivas na amostra quanto ao seu objetivo. Uma delas tratou da avaliação do desenvolvimento da criança, prévia à entrevista, para adequação das questões à capacidade de compreensão do entrevistado; e outra investigou especificamente o uso do termo "toque" pelas crianças como sinônimo de contatos corporais, vez que esse termo é recorrentemente utilizado por entrevistadores em suas interações com as crianças (Powell, Wilson, \& Hasty, 2002; Teoh, Pipe, Johnson, \& Lamb, 2014).

\section{Considerações Finais}

Pesquisas conduzidas na década de 90 identificaram um contínuo de risco de contaminação das repostas das crianças durante entrevistas. As questões abertas ou solicitações estariam no polo em que se constatava menos risco de indução; em seguida apareciam questões focadas, porém ainda abertas; passando-se às questões sobre detalhes específicos ou de múltipla-escolha; às questões de escolha forçada e, por fim, chegando-se às perguntas coercitivas ou 
enganosas (Hershkowitz, 2002). Os estudos recuperados nesta revisão, que abrangeu toda a década seguinte, consolidaram a existência deste contínuo e acrescentaram informações importantes aos profissionais da área, por exemplo, no que diz respeito à importância dos facilitadores (Hershkowitz, 2002).

Questões abertas e facilitadores favoreceram relatos mais detalhados tanto em entrevistas sem uso de qualquer protocolo quanto em entrevistas com protocolos, como o NICHD e o RATAC. Por outro lado, questões diretivas, de múltipla-escolha e sugestivas favoreceram contradições nos relatos, o que colocou em cheque a veracidade das informações prestadas. Isso foi ainda mais significativo com relação às questões de múltipla-escolha e sugestivas na presença das quais as crianças e adolescentes apresentaram contradições inclusive sobre detalhes centrais do suposto abuso.

$\mathrm{O}$ uso de protocolo - principalmente o NICHD que foi o mais pesquisado na amostra - atenuou questões de gênero, favoreceu a análise com maior precisão sobre a credibilidade do relato das crianças, diminuiu a incidência de questões sugestivas e de múltipla-escolha e aumentou a taxa de questões abertas. Assim, pode-se afirmar que o uso de protocolo favoreceu a adesão a boas práticas, o que não foi observado de forma tão significativa com as orientações do Memorando de Boas Práticas já que, quando este foi o padrão adotado, houve poucas perguntas abertas nas entrevistas.

A quantidade significativa ( $35 \%$ da amostra) de estudos nos quais as técnicas de entrevista utilizadas não foram estruturadas ou não havia qualquer informação sobre o uso de formato padrão para sua condução, é preocupante. Isto porque sinaliza que, mesmo diante de dados consistentes de que formatos estruturados favorecem a adesão a boas práticas, é comum que os profissionais questionem a criança de modo variável, incorrendo com maior facilidade no uso de questões inapropriadas ou em práticas questionáveis.

Quanto ao uso de recursos adicionais à entrevista, o desenho mostrou-se útil ao favorecimento de relatos mais organizados e sequenciais, principalmente, após aplicação do protocolo NICHD. O diagrama corporal, igualmente, foi mais pesquisado como recurso adicional à entrevista com o protocolo NICHD, condição na qual favoreceu a obtenção de informações relevantes, sendo especialmente útil com crianças menores. Entretanto, uma ressalva quanto ao diagrama corporal, é o fato de que este recurso usualmente é utilizado com a formulação de questões múltipla-escolha, que não são favoráveis à fidedignidade dos relatos.

Alguns temas que carecem de maior investimento científico são a técnica de restabelecimento de contexto mental versus físico e o procedimento de avaliação prévia à entrevista sobre o desenvolvimento de repertório verbal e de 
conceitos de tempo, número, cor e espaço pela criança. Além disso, atentando-se à escassez de estudos brasileiros sobre entrevistas com crianças e adolescentes em caso de abuso sexual, sugerem-se novas pesquisas que avaliem a forma como crianças brasileiras referem-se a contatos corporais e respondem aos tipos de enunciados formulados pelos entrevistadores, já que questões de linguagem e culturais podem exercer significativa influência.

Por fim, levantamentos epidemiológicos demonstram que a maior parte dos casos de violência sexual são perpetrados por pessoas próximas às crianças como pais e outros familiares, ou alguém do convívio da criança e do adolescente (Brasil, 2012). Grande parte das pesquisas recuperadas envolveu episódios únicos de abuso supostamente praticado por terceiros, de modo que persiste a demanda de ulteriores investigações sobre abuso intrafamiliar. Subsiste ainda a demanda por estudos com crianças ou adolescentes que passaram por múltiplas situações de abuso, pois dados de pesquisa dão nota de que o relato desse tipo de abuso contém características específicas (Katz et al., 2014).

\section{Referências}

Ahern, E. C., Hershkowitz, I., Lamb, M. E., Blasbalg, U. \& Winstanley, A. (2014). Support and reluctance in the pre-substantive phase of alleged child abuse victim investigative interviews: revised versus standard NICHD protocols. Behavioral Sciences \& The Law, 32(6), 762-774. DOI: 10.1002/bsl.2149.

Aldridge, J., Lamb, M. E., Sternberg, K. J., Orbach, Y., Esplin, P. W. \& Bowler, L. (2004). Using a human figure drawing to elicit information from alleged victims of child sexual abuse. Journal of Consulting and Clinical Psychology, 72(2), 304-316. DOI: 10.1037/0022-006X.72.2.304.

Anderson, G. D., Anderson, J. N. \& Gilgun, J. F. (2014). The influence of narrative practice techniques on child behaviors in forensic interviews. Journal of Child Sexual Abuse, 23(6), 615-34. DOI: 10.1080/10538712.2014.932878.

Blefari, C. A. (2014). Capacitação de profissionais para o uso do protocolo NICHD em entrevistas investigativas de abuso sexual infantil. Dissertação de mestrado, Universidade Tuiuti do Paraná, Curitiba, PR, Brasil. 
Brasil (2012). Abuso sexual é o $2^{\circ}$ tipo de violência mais comum contra crianças, mostra pesquisa. Recuperado em 20 de junho de 2015 de: http://www.brasil.gov.br/saude/2012/05/abuso-sexual-e-o-segundo-maiortipo-de-violencia-contra-criancas-mostra-pesquisa. BRASIL.

Brino, R. F. (2007). Professores como agentes de proteção ao abuso sexual infantil: avaliação de um programa de capacitação. Tese de doutorado, Universidade Federal de São Carlos, São Carlos, SP, Brasil.

Brown, D. A., Lamb, M. E., Lewis, C., Pipe, M.-E., Orbach, Y. \& Wolfman, M. (2013). The NICHD investigative interview protocol: an analogue study. Journal of Experimental Psychology, 19(4), 367-82. DOI: 10.1037/a0035143.

Brubacher, S. P. \& La Rooy, D. (2014). Witness recall across repeated interviews in a case of repeated abuse. Child Abuse \& Neglect, 38(2), 202211. http://dx.doi.org/10.1016/j.chiabu.2013.06.010

Cederborg, A. C., Danielsson, H., La Rooy, D. \& Lamb, M. E. (2009). Repetition of contaminating question types when children and youths with intellectual disabilities are interviewed. Journal of Intellectual Disability Research, 53(5), 440-449. DOI: 10.1111/j.1365-2788.2009.01160.x.

Cheung, M. (2008). Promoting effective interviewing of sexually abused children: A pilot study. Research on Social Work Practice, 18(2), 137-143. DOI: $10.1177 / 1049731507304359$.

Costa, A., \& Zoltowski, A. P. C. (2014). Como escrever um artigo de revisão sistemática. In: S. H. Koller, M. C. P. Couto, J. V. Hohendorff. Manual de produção científica. (pp. 55-70). Porto Alegre: Penso.

Cronch, L. E., Viljoen, J. L. \& Hansen, D. J. (2006). Forensic interviewing in child sexual abuse cases: current techniques and future directions.

Aggression and Violent Behavior, 11, 195-207. DOI:

10.1016/j.avb.2005.07.009.

Darwinkel, E. C., Powell, M. B. \& Sharman, S. J. (2014). Does extensive free narrative prompting minimize the effect of mental reinstatement on children's recall of events? Psychiatry, Psychology and Law, 21(3), 351359. DOI: $\quad 10.1080 / 13218719.2013 .817290$.

Daviesl, G. M., Westcott, H. L. \& Horan, N. (2000). The impact of questioning style on the content of investigative interviews with suspected child sexual 
abuse victims. Psychology, Crime \& Law, 6(2), 81-97. DOI: 10.1080/10683160008410834.

Feltis, B. B., Powell, M. B., Snow, P. C. \& Hughes-Scholes, C. H. (2010). An examination of the association between interviewer question type and story-grammar detail in child witness interviews about abuse. Child Abuse \& Neglect, 34(6), 407-413. DOI: 10.1016/j.chiabu.2009.09.019

Fogarty, K., Augoustinos, M. \& Kettler, L. (2013). Re-thinking rapport through the lens of progressivity in investigative interviews into child sexual abuse. Discourse Studies, 15(4), 395-420. DOI:

10.1177/1461445613482429.

Gava, L., Pelisoli, C. \& Dell’Aglio, D. D. (2013). A perícia psicológica em casos de suspeita de abuso sexual infanto-juvenil. Avaliação Psicológica, 12(2), 137-145.

Gilstrap, L. L. (2004). A missing link in suggestibility research: What is known about the behavior of field interviewers in unstructured interviews with young children? Journal of Experimental Psychology-Applied,10(1), 1324. DOI: 10.1037/1076-898X.10.1.13.

Hershkowitz, I. \& Terner, A. (2007). The effects of repeated interviewing on children's forensic statements of sexual abuse. Applied Cognitive Psychology, 21(9), 1131-1143. DOI: 10.1002/acp.1319.

Hershkowitz, I. (2002). The role of facilitative prompts in interviews of alleged sex abuse victims. Legal and Criminological Psychology, 7(1), 63-71. DOI: $10.1348 / 135532502168388$.

Hershkowitz, I., Fisher, S., Lamb, M. E. \& Horowitz, D. (2007). Improving credibility assessment in child sexual abuse allegations: the role of the NICHD investigative interview protocol. Child Abuse \& Neglect, 31, 99110. DOI: 10.1016/j.chiabu.2006.09.005.

Hershkowitz, I., Orbach, Y., Lamb, M. E., Sternberg, K. J. \& Horowitz, D. (2006). Dynamics of forensic interviews with suspected abuse victims who do not disclose abuse. Child Abuse \& Neglect, 30(7), 753-769. doi:10.1016/j.chiabu.2005.10.016.

Hershkowitz, I., Orbach, Y., Lamb, M. E., Sternberg, K. J. \& Horowitz D. (2002). A comparison of mental and physical context reinstatement in 
forensic interviews with alleged victims of sexual abuse. Applied Cognitive Psychology, 16(4), 429-441. DOI: 10.1002/acp.804.

Kask, K. (2012). Dynamics in using different question types in Estonian police interviews of children. Applied Cognitive Psychology, 26(2), 324-329.

DOI: 10.1002/acp.1831.

Katz, C. \& Hamama, L. (2013). Draw me everything that happened to you: exploring children's drawings of sexual abuse. Children and Youth Services Review, 35(5), 877-882.

http://dx.doi.org/10.1016/j.childyouth.2013.02.007.

Katz, C. \& Hershkowitz, I. (2010). The effects of drawing on children's accounts of sexual abuse. Child Maltreatment, 15(2), 171-179. DOI: 10.1177/1077559509351742.

Katz, C. \& Hershkowitz, I. (2012). The effect of multipart prompts on children's testimonies in sexual abuse investigations. Child Abuse \& Neglect, 36(11), 753-759. http://dx.doi.org/10.1016/j.chiabu.2012.07.002.

Katz, C. \& Hershkowitz, I. (2013). Repeated interviews with children who are the alleged victims of sexual abuse. Research on Social Work Practiceactor, 23(2), 210-218. DOI: 10.1177/1049731512467511.

Katz, C., Barnetz, Z. \& Hershkowitz, I. (2014). The effect of drawing on children's experiences of investigations following alleged child abuse. Child Abuse \& Neglect, 38(5), 858-867. http://dx.doi.org/10.1016/j.chiabu.2014.01.003.

Korkman, J., Santtila, P. \& Sandnabba, N. K. (2006). Dynamics of verbal interaction between interviewer and child in interviews with alleged victims of child sexual abuse. Scandinavian Journal of Psychology, 47(2), 109-119. DOI: 10.1111/j.1467-9450.2006.00498.x.

Korkman, J., Santtila, P., Westeraker, M. \& Sandnabba, N. K. (2008). Interviewing techniques and follow-up questions in child sexual abuse interviews. European Journal of Developmental Psychology, 5(1), 108128. http://dx.doi.org/10.1080/17405620701210460.

Lamb, M. E. \& Fauchier, A. (2001). The effects of question type on selfcontradictions by children in the course of forensic interviews. Applied Cognitive Psychology, 15(5), 483-491. DOI: 10.1002/acp.726. 
Lamb, M. E. \& Garretson, M. E (2003). The effects of interviewer gender and child gender on the informativeness of alleged child sexual abuse victims in forensic interviews. Law and Human Behavior, 27(2), 157-171. DOI: 10.1023/A:1022595129689.

Lamb, M. E., Hershkowitz, I., Orbach, Y. \& Esplin, P. W. (2008). Tell me what happened: Structured investigative of child victims and witnesses. Chichester: Wiley-Blackewell. Oxford, UK. DOI: 10.1002/9780470773291.fmatter.

Lamb, M. E., Hershkowitz, I., Sternberg, K. J., Esplin, P. W., Hovav, M., Manor, T. \& Yudilevitch, L. (1996). Effects of investigative utterance types on Israeli children's responses. International Journal of Behavioral Development, 19(3), 627-637. DOI: 10.1177/016502549601900310.

Lamb, M. E., Orbach, Y., Sternberg, K. J., Aldridge, J., Pearson, S., Stewart, H. L., Esplin P. W. \& Bowler, L. (2009). Use of a structured investigative protocol enhances the quality of investigative interviews with alleged victims of child sexual abuse in britain. Applied Cognitive Psychology, 23(4), 449-467. DOI: 10.1002/acp.1489.

Lamb, M. E., Stemberg, K. J., Orbach, Y., Esplin, P. W., Stewart, H. \& Mitchell, S. (2003). Age differences in young children's responses to openended invitations in the course of forensic interviews. Journal of Consulting and Clinical Psychology, 71(5), 926-934. DOI: 10.1037/0022006X.71.5.926.

Lindberg, M. A., Chapman, M. T., Samsock, D., Thomas, S. W. \&Lindberg, A. W. (2003). Comparisons of three different investigative interview techniques with young children. Journal of Genetic Psychology, 164(1), 528. DOI: $10.1080 / 00221320309597500$.

Lyon, T. D. (2012). Twenty-five years of interviewing research and practice: dolls, diagrams, and the dynamics of abuse disclosure. APSAC Advisor, 24(1-2), 14-19.

Orbach, Y. \& Lamb, M. E. (2000). Enhancing children's narratives in investigative interviews. Child Abuse \& Neglect, 24(12), 1631-1648. DOI: 10.1016/S0145-2134(00)00207-6.

Orbach, Y. \& Lamb, M. E. (2001). The relationship between within-interview contradictions and eliciting interviewer utterances. Child Abuse \& Neglect, 25(3), 323-333. http://doi.org/10.1016/S0145-2134(00)00254-4. 
Orbach, Y., Hershkowitz, I., Lamb, M. E., Sternberg, K. J., Esplin, P. W. \& Horowitz, D. (2000). Assessing the value of structured protocols for forensic interviews of alleged child abuse victims. Child Abuse \& Neglect, 24(6), 733-752. http://doi.org/10.1016/S0145-2134(00)00137-X.

Patterson, T. \& Pipeb, M. E. (2009). Exploratory assessments of child abuse: Children's responses to interviewer's questions across multiple interview sessions. Child Abuse \& Neglect, 33(8), 490-504. DOI:

10.1016/j.chiabu.2008.12.012.

Phillips, E., Oxburgh, G., Gavin, A. \& Myklebust, T. (2012). Investigative interviews with victims of child sexual abuse: The relationship between question type and investigation relevant information. Journal of Police and Criminal Psychology, 27(1), 45-54. DOI: 10.1007/s11896-011-9093$\mathrm{z}$.

Plano Nacional de Enfrentamento da Violência Sexual contra Crianças e Adolescentes: Faça bonito. (2013). Recuperado em 15 de junho de 2015 de: http://www.comitenacional.org.br/.

Poole, D. A. \& Bruck, M. (2012). Divining testimony? The impact of interviewing props on children's reports of touching. Developmental Review, 32(3), 165-180. ～http://doi.org/10.1016/j.dr.2012.06.007.

Poole, D. A. \& Dickinson, J. J. (2011). Evidence supporting restrictions on uses of body diagrams in forensic interviews. Child Abuse \& Neglect, 35(9), 659-669. ～http://doi.org/10.1016/j.chiabu.2011.05.004.

Powell, M. B., Wilson, J. C. \& Hasty, M. K. (2002). Evaluation of the usefulness of "Marvin": A computerized assessment tool for investigative interviewers of children. Computers in Human Behavior, 18(5), 577-592. http://doi.org/10.1016/S0747-5632(02)00003-1.

Santila, P., Korkman, J. \& Sandnabba, N. K. (2004). Effects of interview phase, repeated interviewing, presence of a support person, and anatomically detailed dolls on child sexual abuse interviews. Psychology, Crime \& Law, 10(1), 21-35. http://dx.doi.org/10.1080/1068316021000044365.

Schaefer, L. S., Rossetto, S. \& Kristensen, C. H. (2012). Perícia psicológica no abuso sexual de crianças e adolescentes. Psicologia: Teoria e Pesquisa, 28(2), 227-234. 
Schreiber, N. (2000). Interviewing techniques in sexual abuse cases - a comparison of a day-care abuse case with normal abuse cases. Swiss Journal of Psychology, 59(3), 196-206. DOI: 10.1024//14210185.59.3.196.

Snow, P. C., Powell, M. B. \& Murfett, R. (2009). Getting the story from child witnesses: Exploring the application of a story grammar framework. Psychology, Crime \& Law, 15(6), 555-568. DOI: 10.1080/10683160802409347.

Souza, C. J. (2014). Psicologia Jurídica: encontros e desencontros em sua prática. Recuperado em 10 de julho de 2015 de: http://www.tjdft.jus.br/institucional/imprensa/artigos/2014/psicologiajuridica-encontros-e-desencontros-em-sua-pratica-servidora-cristianajobim-souza.

Sternberg, K. J., Lamb, M. E., Orbach, Y., Esplin, P. W. \& Mitchell, S. (2001). Use of a structured investigative protocol enhances young children's responses to free-recall prompts in the course of forensic interviews. Journal of Applied Psychology, 86(5), 997-1005. DOI: 10.1037//00219010.86.5.997.

Stracke, C. B. (2013). Avaliação da estrutura da entrevista investigativa com crianças. Dissertação de mestrado, Pontifícia Universidade Católica de Rio Grande do Sul. Porto Alegre, RS, Brasil.

Teoh, Y. S. \& Lamb, M. E. (2010). Preparing children for investigative interviews: Rapport-building, instruction, and evaluation. Applied Developmental Science, 14(3), 154-163. http://dx.doi.org/10.1080/10888691.2010.494463.

Teoh, Y. S., Pipe, M. E., Johnson, Z. H. \& Lamb, M. (2014). Eliciting accounts of alleged child sexual abuse: How do children report touch? Journal of Child Sexual Abuse, 23(7), 792-803. http://dx.doi.org/10.1080/10538712.2014.950400.

Teoh, Y. S., Yang, P. J., Lamb, M. E. \& Larsson, A. S. (2010). Do humans figure diagrams help alleged victims of sexual abuse provide elaborate and clear accounts of physical contact with alleged perpetrators? Applied Cognitive Psychology, 24(2), 287-300. DOI: 10.1002/acp.1564.

Westcott, H. L. \& Kynan, S. (2004). The application of a 'story-telling' framework to investigative interviews for suspected child sexual abuse. 
Legal and Criminological Psychology, 9(1), 37-56. DOI:

10.1348/135532504322776843.

Williams, L. C. A., Hackbarth, C., Blefari, C. A., Padilha, M. G. S. \& Peixoto, C. E. (2014). Investigação de suspeita de abuso sexual infanto-juvenil: O protocolo NICHD. Temas em Psicologia, 22(2), 1-18.

World Health Organization - WHO. (1999). WHO Consultation on Child Abuse Prevention. Geneva: WHO. Recuperado em 12 de julho de 2015 de: http://www.who.int/iris/handle/10665/65900. 